\title{
Creative Mathematics Education
}

\author{
Edgar E. Escultura \\ G.V.P. College of Engineering, J. Nehru Technical University, Visakhapatnam, India \\ Email: escultur36@yahoo.com
}

Received November $1^{\text {st }}, 2011$; revised December $7^{\text {th }}, 2011$; accepted December $19^{\text {th }}, 2011$

\begin{abstract}
Creativity and critical thinking are the core values of science. Since mathematics is its primary language, the student of mathematics must imbibe and consolidate them. Critical thinking is consolidated in the critique of current mathematics and its foundations, creativity in the construction of a mathematical space or system. Therefore, the student of mathematics must go through the twists and turns of the critique-rectification of current mathematics and its foundations which in this paper focuses on the real and complex number systems that results in the construction of the contradiction-free new real number system and the complex vector plane. Since this is an expository paper on creative education much of the content is quoted from the Author's previous works.
\end{abstract}

Keywords: Adjacent Decimals; Axioms; Banach-Tarski Paradox; Russell Paradox; Creativity; Critical Thinking; Dark Number; Decimal Integer; Goldbach's Conjecture; G-Limit; G-Norm G-Sequnce; Lexicographic Ordering; Recurring 9s; Self-Reference; Vacuous Concept; Fermat's Last Theorem

\section{Introduction}

We introduce and develop mathematics as primary language of science. A mathematical space is the language of a particular branch of science. Its vocabulary consists of words and symbols called concepts whose usage is defined by its axioms or basic premises, e.g., the real number system has the concepts $0, \pi,+$ and $\times$. The concepts + and $\times$ are called the additive and multiplicative operations, respectively, defined by its field axioms (Royden, 1983).

In creative mathematics education the student is guided through and involved in actual construction of a mathematical space, introduction of concepts, statement, formulation and use of axioms and proofs of conclusions from them called theorems at the appropriate level. Since a mathematical space is defined solely by its axioms, two distinct mathematical systems are independent and a concept in one is not defined (ill-defined, ambiguous, nonsense) in the other. Therefore, the rules of inference rest solely on the axioms. In particular, formal logic is not valid for mathematical reasoning because it has nothing to do with the axioms (Escultura, 2009a). Part of the requirements for effective mathematics education is that the subjects or courses are consistent and its axioms are adequate; otherwise, research grinds to a halt and learning is limited. The same applies to science.

\section{Departure from Traditions}

In traditional mathematics the concepts are supposed to be concepts of individual thought which are ambiguous being inaccessible to others and can neither be studied collectively nor axiomatized. Therefore, they cannot be the subject matter of mathematics, so noted David Hilbert a century ago. Hence he proposed to admit as concepts only objects in the real world such as word, symbol and figure that everyone can study subject to consistent axioms. However, this important advance for mathematics has not been grasped by mathematicians in general, e.g., the popularly accepted equation, $1=0.99 \cdots$, is false because the left and right terms are different objects. It is akin to saying apple $=$ orange.

We identify some ambiguity that must be avoided as it is a source of contradiction or paradox.

1) Self-reference. (The barber paradox) The barber of Seville shaves those and only those who do not shave themselves. Who shaves the barber? The Russell paradoxes belong to this category; so does the indirect proof. Here is a famous Russell paradox:

Let $\mathrm{M}$ be the set of all sets where each element does not belong to itself, i.e. $M=\{m: m \notin m\}$. Either $M \in M$ or $M \notin M$. If $\mathrm{M} \in \mathrm{M}$, the defining statement of $\mathrm{M}$ holds and $\mathrm{M} \notin \mathrm{M}$. However, if $\mathrm{M} \notin \mathrm{M}$, then $\mathrm{M}$ satisfies the defining condition; therefore $M \in M$. The proof is self-referent: a set is defined by its elements and each element is defined by its membership in the set (Lakshmikantham et al., 2009). In general we call self-referent any proposition where the hypothesis follows from or is negated or simply referred to by the conclusion.

2) Vacuous concept or proposition. Consider the concept i called imaginary number defined by the statement, the root or solution of the equation,

$$
x^{2}+1=0
$$

among real numbers. Obviously, this concept which has been traditionally denoted by $\mathrm{i}=\sqrt{(-1)}$ is vacuous since Equation (1) has no solution. Consequently, we have,

$$
\mathrm{i}=\sqrt{(-1)}=\sqrt{(1 /-1)}=1 / \mathrm{i}=-\mathrm{i} \text {. }
$$

Clearly, Equation (2) yields $\mathrm{i}=0$ and $1=0$ and, for any real number $r, r=0$ and both the real number and complex number systems collapse (see remedy in Escultura, in press).

Another example is Perron paradox (Young, Mathematicians, 1980): let $\mathrm{N}$ be the largest integer. Then, one and only one of 
the following holds: $\mathrm{N}<1, \mathrm{~N}=1, \mathrm{~N}>1$. Obviously, the first inequality is false. The third statement cannot be true, otherwise, we will $\mathrm{N}^{2}>\mathrm{N}$ contradicting the definition of $\mathrm{N}$. Therefore, $\mathrm{N}$ $=1$ which is clearly false. What is the culprit here? $\mathrm{N}$ is vacuous.

3) Infinity is any concept with this attribute, e.g., infinite set and nonterminating decimal. Such concept is ambiguous. An infinite set is the negation of a finite set whose elements can be put into $1-1$ correspondence with a segment of the integers, i.e., the set of integers between two known integers. Unlike infinite set, all its members are known or identifiable. In other words, an infinite set cannot be contained in a finite set, i.e., some element is in the complement. Another example is nonterminating decimal since not all its digits are known. This ambiguity is inherent and cannot be removed. It may be contained by approximating it by certainty. For example, a nonterminating decimal is approximated by a terminating decimal, which has no ambiguity since every digit is known, within desired margin of error.

Any categorical statement about ambiguous set is ambiguous. An example is one involving the universal or existential quantifier (i.e., every or there exists). Suppose we want to verify that every element of a given infinite set has property P. We start with an element $\mathrm{x}$, if possible. Assuming $\mathrm{x}$ has property $\mathrm{P}$, we proceed to another element, and to another, etc. Clearly, this verification cannot be completed which is an ambiguity. The same holds true of the existential quantifier.

4) Large and small numbers. Scientists introduced order of magnitude to deal with such numbers (Escultura, 2009b). They are numbers of the form $\mathrm{a} \times 10^{\mathrm{n}}$, where $\mathrm{a}$ is one of the digits or basic integers, $0,1, \cdots, 9$. The order of magnitude here is $10^{\mathrm{n}}$. Similarly, quantum physicists write the mass of the neutrino as $\eta=8.5 \times 10^{-8}$ amu (Escultura, 2009c) the order of magnitude being $10^{-8}$. Some principles of physics, e.g., Heisenberg uncertainty principle (Escultura, Pillars, 2007), follow from the ambiguity of large and small numbers. (For more on ambiguity see Kline, 1980; Lakatos, 1976) This level of mathematical precision is necessary for the training of a mathematician at the appropriate level to imbibe the core values of mathematics and science, namely, creativity and critical thinking. This, again, is a departure from traditional mathematics and the antidote to rote learning and mechanical thinking (e.g., computation and manipulation of symbols). Creativity is acquired in the course of constructing a mathematical space and critical thinking through critique-rectification of mathematics. It is the latter that enables the mathematician or scientist to spot errors in and inadequacy of mathematics and science and paves the way for their advancement. To consolidate creativity and critical thinking among mathematics and science students, we get them involved in the critique-rectification of the real and complex number systems to remedy their defects along with the construction of the new real number system and complex vector plane (Escultura, 2009b).

Another tradition to avoid in mathematics is admission of undefined concepts in constructing a mathematical space which introduces ambiguity. While they may be admitted initially, the choice of the axioms is not complete until every concept is defined. The choice of axioms depends on what the mathematical space is for. It is different in science where the axioms are natural laws and beyond the choice or control of the scientist.

\section{Critique of the Real Number System}

We summarize the full critique-rectification of foundations and the real number system.

1) Since the union of countable sets is countable no known construction of nondenumerable set exists and Cantor's diagonal method is flawed and fails to prove the nondenumerability of the real numbers.

2) Since infinite set is ambiguous, the concept limit of calculus is ambiguous and all completeness and compactness theorems involving infinite set of real numbers collapse. The appropriate remedy for this concept is introduction of the g-norm (Escultura, 2009b).

3) The field axioms of the real number system are inconsistent, counterexamples to the trichotomy and completeness axioms having been found (Escultura, 2009b). Among their implications are:

The real number system is not linearly ordered by the relation "<" which also collapses analytic geometry. However, the trichotomy axiom is true in the new real number system and follows from its lexicographic ordering by " $<$ " which linearly orders it (Escultura, 2009b).

4) The only defined real numbers are the terminating decimals and fractions with denominator 2 or 5 . Nonterminating decimals are not defined. The traditional definition of irrational number as nonterminating nonperiodic decimal is ambiguous because this behavior is unverifiable.

\section{The New Real Number System}

The critique-rectification of the real number system yielded a much simpler construction of the new real number system $\mathbf{R}^{*}$ (Escultura, 2009b) that contains the real number system $\mathbf{R}$ as its proper subspace.

\section{The Axioms and Terminating Decimals}

Axiom 1. R* contains the elements 0,1 called the additive and multiplicative identities, respectively.

Their properties are defined by the addition and multiplication tables below. First, we define the digits or basic integers, 0 , $1,2,3,4,5,6,7,8,9$, as follows:

$$
\begin{aligned}
& 2=1+1, \quad 3=2+1, \quad 4=3+1, \quad 5=4+1, \\
& 6=5+1, \quad 7=6+1, \quad 8=7+1, \quad 9=8+1 .
\end{aligned}
$$

We define their extensions to the integers as follows: $10=9$ $+1,11=10+1$, etc., which we write in scientific notation later.

Axiom 2. The addition table below defines the sums of basic

\begin{tabular}{|c|c|c|c|c|c|c|c|c|c|c|}
\hline+ & 0 & 1 & 2 & 3 & 4 & 5 & 6 & 7 & 8 & 9 \\
\hline 0 & 0 & 1 & 2 & 3 & 4 & 5 & 6 & 7 & 8 & 9 \\
\hline 1 & 1 & 2 & 3 & 4 & 5 & 6 & 7 & 8 & 9 & \\
\hline 2 & 2 & 3 & 4 & 5 & 6 & 7 & 9 & 9 & & \\
\hline 3 & 3 & 4 & 5 & 6 & 7 & 8 & 9 & & & \\
\hline 4 & 4 & 5 & 6 & 7 & 8 & 9 & & & & \\
\hline 5 & 5 & 6 & 7 & 9 & 9 & & & & & \\
\hline 6 & 6 & 7 & 8 & 9 & & & & & & \\
\hline 7 & 7 & 8 & 9 & & & & & & & \\
\hline 8 & 8 & 9 & & & & & & & & \\
\hline 9 & 9 & & & & & & & & & \\
\hline
\end{tabular}
integers that are basic integers. 
The extension of this table to sums of integers is obvious.

Axiom 3. The multiplication table below defines the products of basic integers that are basic integers.

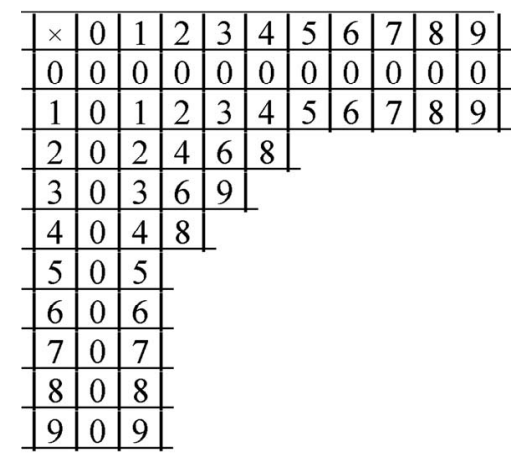

Its extension to products of integers is likewise obvious.

They are, in fact, the same addition and multiplication tables in the real number system and their extensions apply to welldefined fractions and terminating decimals. Therefore, their properties are retained in $\mathbf{R}^{*}$. The only difference is: they do not apply to nonterminating decimals which are not defined in the real number system; we shall define them in $\mathbf{R}^{*}$.

We now introduce and define the inverses. The additive inverse of an integer $\mathrm{x}$, denoted by $-\mathrm{x}$, satisfies the equation,

$$
\mathrm{x}+(-\mathrm{x})=0 .
$$

The difference between integers $\mathrm{x}$ and $\mathrm{y}$, denoted by $\mathrm{x}-\mathrm{y}$, is defined as $x+-y$. This operation is called subtraction, the inverse of addition.

To avoid confusion, we may write the product of two integers $a$ and $b$ by $a(b)$ or $a b$. The multiplicative inverse of a nonzero integer $\mathrm{x}$, denoted by $1 / \mathrm{x}$, satisfies,

$$
\mathrm{x}(1 / \mathrm{x})=1 \text {. }
$$

The quotient of two integers $x$ and $y$, denoted by $\mathrm{x} \div \mathrm{y}$, where $y$ has no prime factor other than 2 or 5 , is a number $z$ that satisfies the equation, $x=y z$. We also write this quotient as the fraction $\mathrm{x} / \mathrm{y}$ and when the division is carried out the result is a terminating decimal.

In scientific notation we write the rest of the integers as base 10 place-value numerals:

$$
a_{n} a_{n-1} \cdots a_{1}=a_{n} 10^{n}+a_{n-1} 10^{n-1}+\cdots+a_{1},
$$

where the $a_{n} s$ are basic integers. We define a terminating decimal as follows:

$$
\begin{aligned}
& a_{n} a_{n-1} \cdots a_{1} \cdot b_{k} b_{k-1} \cdots b_{1} \\
= & a_{n} 10^{n}+a_{n-1} 10^{n-1}+\cdots+a_{1} \\
& +b_{1} / 10+b_{2} / 10^{2}+\cdots+b_{k} / 10^{k} \\
= & a_{n} 10^{n}+a_{n-1} 10^{n-1}+\cdots+a_{1} \\
& +b_{1}(0.1)+b_{2}(0.1)^{2}+\cdots+b_{k}(0.1)^{k} .
\end{aligned}
$$

where $a_{n} a_{n-1} \cdots a_{1}$ is the integral part, $b_{1} b_{2} \cdots b_{k}$ the decimal part and $0.1=1 / 10$. The terminating decimals are well-defined since the reciprocal of 10 has only the factors 2 and 5. If $x$ and $\mathrm{y}$ are relatively prime integers, i.e., they have no common prime divisor, $\mathrm{y} \neq 0$, then the quotient $\mathrm{x} / \mathrm{y}$ of $\mathrm{x}$ by $\mathrm{y}$ exists only if $\mathrm{y}$ has no prime factor other than 2 or 5.

\section{The Nonterminating Decimals}

A sequence of terminating decimals of the form,

$$
\text { N.a } a_{1}, \text { N.a } a_{1} a_{2}, \cdots, N . a_{1} a_{2} \cdots a_{n}, \cdots,
$$

where $\mathrm{N}$ is an integer, $\mathrm{a}_{\mathrm{n}}$ basic integer and there is a rule for choosing each $a_{n}$, is called standard generating or g-sequence. Its nth g-term, N. $\mathrm{a}_{1} \mathrm{a}_{2} \cdots \mathrm{a}_{\mathrm{n}}$, a terminating decimal, approximates its nonterminating) g-limit

$$
\text { N. } a_{1} a_{2} \cdots a_{n}, \cdots,
$$

at margin of error $10^{-n}$ provided each nth g-term is computable (there is some algorithm for determining or computing the nth digit). For example, the digits of $\pi$ can be computed from its infinite series expansion. A decimal is normal if every digit is chosen at random from the digits (Escultura, 2009b). The g-limit of (10) is the nonterminating decimal (11) provided the nth digits are not all 0 beyond a value of $n$. In this case, we say that the g-sequence (10) converges to the nonterminating decimal (11) in the g-norm where the g-norm of a decimal is itself. Otherwise, (11) is terminating. A decimal consists of the integral part, the integer to the left of the decimal point, and the decimal part, the sequence of digits to the right of the decimal point. The integers are ill-defined by the field axioms of the real number system but defined in the new real number system by Axioms 1, 2 and 3 as the integral parts of the decimals.

In the real number system the rationals coincide with the terminating decimals which are periodic while an irrational number is nonterminating nonperiodic. Both properties of the latter are unverifiable. Thus, this concept is ill-defined, ambiguous and is neither a decimal nor a new real number.

We define the nth distance $d_{n}$ between two decimals $a, b$ as the numerical value of the difference between their nth g-terms, $a_{n}, b_{n}$, i.e., $d_{n}=\left|a_{n}-b_{n}\right|$ and their g-distance is the g-limit of $\mathrm{d}_{\mathrm{n}}$ We denote the g-closure of $\mathbf{R}$ by $\mathbf{R}^{*}$, the new real number system.

The nth g-term of a nonterminating decimal repeats every preceding digit at the same order so that if finite terms are deleted the nth g-term and g-limit are unaltered and the remaining terms also comprise its g-sequence. Thus, a nonterminating decimal has many g-sequences that belong to the equivalence class of its g-limit.

Since addition and multiplication and their inverse operations, subtraction and division, are defined only on terminating decimals computing nonterminating decimals is done by approximating each term or factor by its nth g-term (n-truncation), a terminating decimal, and using their approximations to find the nth g-term of its sum or product. The same approximation holds for the difference and quotient (if defined). Thus, we have retained standard computation but avoided the ambiguity, contradictions and paradoxes of the real number system. We have also avoided vacuous approximation because nonterminating decimals are g-limits of their g-sequences which exist and belong to $\mathbf{R}^{*}$. Moreover, we have contained the ambiguity of nonterminating decimals by approximating them by their nth $\mathrm{g}$ terms.

As we raise $n$, the tail digits of the nth g-term of any decimal recedes to the right indefinitely, i.e., it becomes steadily smaller until it is unidentifiable from the tail digits of the rest of the decimals. While it tends to 0 in the standard norm it never reaches 0 in the g-norm since the tail digits are never all equal to 0 ; it is also not a decimal since the digits are not fixed. Since 


\section{E. E. ESCULTURA}

none of the tail digits of a decimal is distinguishable from the rest of the tail digits this set cannot be split into two distinct subsets which makes it a continuum in the algebraic sense.

In iterated computation to get closer and closer approximation of a decimal, e.g., calculating $f(n)=\left(2 n^{4}+1\right) / 3 n^{4}, n=1,2, \cdots$, the tail digits may vary but recede to the right indefinitely and become steadily smaller leaving fixed digits behind that define a decimal. We approximate the result by taking its nth g-term to desired margin of error and choosing $n$ suitably.

\section{The Dark Number $\mathrm{d}^{*}$}

Consider the sequence of decimals,

$$
(\delta)^{\mathrm{n}} \mathrm{a}_{1} \mathrm{a}_{3} \cdots \mathrm{a}_{\mathrm{k}}, \mathrm{n}=1,2, \cdots,
$$

where $\delta$ is any of the decimals, $0.1,0.2,0.3, \cdots, 0.9, \mathrm{a}_{1}, \cdots$, $\mathrm{a}_{\mathrm{k}}$, basic integers (not all 0 simultaneously). We call the nonstandard sequence (12) d-sequence. For fixed combination of $\delta$ and the $\mathrm{a}_{\mathrm{j}} \mathrm{s}, \mathrm{j}=1, \cdots, \mathrm{k}$, in (12) the nth d-term is a terminating decimal and as $n$ increases indefinitely it traces the tail digits of some nonterminating decimal and becomes smaller and smaller until it is indistinguishable from the tail digits of the other decimals. As $n \rightarrow \infty$ the nth d-term recedes to the right and tends to some number $\mathrm{d}$, its $\mathrm{d}$-limit in the d-norm, which is never 0 (since the $\mathrm{a}_{\mathrm{j}} \mathrm{s}$ are not 0 simultaneously). It is called dark number $\mathrm{d}$ which is indistinguishable from the rest of the d-limits of (12) for all other choices of $\delta$ and $\mathrm{a}_{\mathrm{j}} \mathrm{s}$. The set of all dark numbers for all choices of $\delta$ and $a_{j} s$ is a countable continuum (in the algebraic sense since no notion of open set is involved) denoted by $\mathrm{d}^{*}$. Thus, $\mathrm{d}^{*}$ is set-valued, countably infinite and a continuum (negation of discrete) of dark numbers; the decimals are joined together by the continuum $\mathrm{d}^{*}$ at their tails. While the nth d-term of (12) becomes smaller and smaller with indefinitely increasing $\mathrm{n}$ it is greater than 0 no matter how large $\mathrm{n}$ is so that if $\mathrm{x}$ is a decimal, $0<\mathrm{d}<\mathrm{x}$. If an equation or function is satisfied by every dark number $d$ we may write $d^{*}$ in place of $d$ so that we can write $0<\mathrm{d}^{*}<\mathrm{x}$ in the above inequality (Escultura, 2008).

Since the tail digits of all the nonterminating decimals form a countable combination of the basic digits $0,1, \cdots, 9$ they are countably infinite, i.e., in one-one correspondence with the integers; we call its cardinality countably infinite. Thus, both the integers and $\mathrm{d}^{*}$ are countably infinite but while the former is discrete, the latter is a continuum. Any set whose elements can be labeled by integers or there is one-one correspondence with the integers is countably infinite. It follows that the countable union of countable set is countable.

Observation. Cantor's diagonal method does not prove the existence of nondenumerable set; the off-diagonal elements generated by his method are countable union of countable sets; therefore, it is countable.

Corollary. Nondenumerable set does not exist.

Thus, the continuum hypothesis of set theory (which is equivalent to the axioms of choice is false. Moreover, it is not necessary to develop set theory as a universal language for mathematics since its axioms are not valid for any other mathematical space.

Like a nonterminating decimal, an element of $\mathrm{d}^{*}$ is unaltered if finite d-terms are altered or deleted from its d-sequence. When $\delta=1$ and $\mathrm{a}_{1} \mathrm{a}_{2} \cdots \mathrm{a}_{\mathrm{k}}=1$ (12) is called the basic or principal d-sequence of $\mathrm{d}^{*}$, its $\mathrm{d}$-limit the basic element of $\mathrm{d}^{*}$; basic because all its d-sequences can be derived from it. The prince- pal d-sequence of $\mathrm{d}^{*}$ is,

$$
(0.1)^{\mathrm{n}}, \mathrm{n}=1,2, \cdots
$$

obtained from the iterated difference,

$$
\begin{aligned}
& \mathrm{N}-(\mathrm{N}-1) .99 \cdots=1-0.99 \cdots=0 \\
& \text { with excess remainder } 0.1 ; \\
& 0.1-0.09 \cdots=0 \\
& \text { with excess remainder } 0.01 ; \\
& 0.01-0.009=0 \\
& \text { with excess remainder } 0.001 ;
\end{aligned}
$$

Taking the nonstandard g-limits of the extreme left and right sides of (14), recalling that the g-limit of a decimal is itself and denoting by $d_{p}$ the $d$-limit of the principal $d$-sequence on the rightmost side we have,

$$
\mathrm{N}-(\mathrm{N}-1) .99 \cdots=1-0.99 \cdots=\mathrm{d}_{\mathrm{p}} .
$$

By convention, since all the elements of $\mathrm{d}^{*}$ share its properties whenever we have a statement "every element $d$ of $\mathrm{d}^{*}$ has property P" we may write " $d$ " has property P", meaning, this statement is true of every element of $\mathrm{d}^{*}$. This applies to any equation involving an element of $\mathrm{d}^{*}$. Therefore,

$$
\mathrm{d}^{*}=\mathrm{N}-(\mathrm{N}-1) .99 \cdots=1-0.99 \cdots \text {. }
$$

Like a decimal, we define the d-norm of $\mathrm{d}^{*}$ as $\mathrm{d}^{*}$ and $\mathrm{d}^{*}>0$. (The dark superstring is qualitatively modeled by $\mathrm{d}^{*}$ (Escultura, 2011).

Theorem. The d-limits of the indefinitely receding to the right $n$th $\mathrm{d}$-terms of $\mathrm{d}^{*}$ is a continuum that coincides with the g-limits of the tail digits of the nonterminating decimals traced by those $n$th $d$-terms as the $\mathrm{a}_{\mathrm{k}} \mathrm{s}$ vary along the basic digits.

If $\mathrm{x}$ is nonzero decimal, terminating or nonterminating, there is no difference between the d-limits of sequences $(0.1)^{\mathrm{n}}$ and $\mathrm{x}(0.1)^{\mathrm{n}}$ as they become indistinguishably small as $\mathrm{n}$ increases indefinitely. This is analogous to the sandwich theorem of calculus that says, $\lim (\mathrm{x} / \sin \mathrm{x})=1$, as $\mathrm{x} \rightarrow 0$; in the proof, it uses the fact that $\sin \mathrm{x}<\mathrm{x}<\tan \mathrm{x}$ or $1<\mathrm{x} / \sin \mathrm{x}<\sec \mathrm{x}$ where both extremes tend to 1 so that the middle term tends to 1 also. In our case, if $0<\mathrm{x}<1,0<\mathrm{x}(0.1)^{\mathrm{n}}<(0.1)^{\mathrm{n}}$ and both extremes tend to 0 so must the middle term and they become indistinguishably small as $\mathrm{n}$ increases indefinitely. If $\mathrm{x}>1$, we simply reverse the inequality and get the same conclusion. Therefore, we have, $x d_{p}=d_{p}\left(\right.$ where $d_{p}$ is the principal element of $\left.d^{*}\right)$ and since the elements of $\mathrm{d}^{*}$ share this property we have $\mathrm{xd}^{*}=\mathrm{d}$. We consider $\mathrm{d}^{*}$ the equivalence class of its elements. In the case of $\mathrm{x}+(0.1)^{\mathrm{n}}$ and $\mathrm{x}$, we look at the nth g-terms of each and, as $\mathrm{n}$ increases indefinitely, $\mathrm{x}+(0.1)^{\mathrm{n}}$ and $\mathrm{x}$ become indistinguishable. Now, since $(0,1)^{\mathrm{n}}>\left((0.1)^{\mathrm{m}}\right)^{\mathrm{n}}>0$ and the extreme terms both tend to 0 as $\mathrm{n}$ increases indefinitely, so must the middle term tend to 0 so that they become indistinguishably small $\left(\mathrm{d}^{*}\right.$ is called dark number for being indistinguishable from 0 yet greater than 0 ). To summarize, if $\mathrm{x}$ is not a decimal integer (a decimal integer has the form, $\mathrm{x}=\mathrm{N} .99 \cdots, \mathrm{N}=0,1, \cdots$ ) then,

$$
\begin{aligned}
& x+d^{*}=x ; \text { otherwise, if } x=N .99 \cdots, \\
& x+d^{*}=N+1, x-d^{*}=x ; \text { if } x \neq 0, d^{*}=d^{*} ; \\
& \left(d^{*}\right)^{n}=d^{*}, n=1,2, \cdots, N=0,1, \cdots ; \\
& 1-d^{*}=0.99 \cdots ; \quad N-(N-1) .99 \cdots ; \\
& 1-0.99 \cdots=d^{*}, \quad N=1,2, \cdots .
\end{aligned}
$$




\section{E. E. ESCULTURA}

Again, it follows that the g-closure of $\mathbf{R}$ is $\mathbf{R}^{*}$; we now include in $\mathbf{R}^{*}$ the additive inverses of elements of $\mathbf{R}^{*}$ and its well-defined multiplicative inverses. We also include in $\mathbf{R}^{*}$ the upper bounds of divergent sequences of terminating decimals and integers called unbounded number $\mathrm{u}^{*}$ (countably infinite). We follow the same convention for $\mathrm{u}^{*}$ : whenever we have a statement " $u$ " has property $P$ for every element $u$ of $u$ ", we simply say " $u$ " has property P). Then $\mathrm{u}^{*}$ satisfies,

$$
x+u^{*}=u^{*} ; \text { for } x \neq 0, x u^{*}=u^{*} .
$$

Neither $\mathrm{d}^{*}$ nor $\mathrm{u}^{*}$ is a decimal; their properties are solely determined by their sequences. Then $\mathrm{d}^{*}$ and $\mathrm{u}^{*}$ have the following dual or reciprocal properties and relationship:

$$
0 \mathrm{~d}^{*}=0,0 / \mathrm{d}^{*}=0,0 \mathrm{u}^{*}=0,0 / \mathrm{u}^{*}=0,1 / \mathrm{d}^{*}=\mathrm{u}^{*}, 1 / \mathrm{u}^{*}=\mathrm{d}^{*}
$$

Numbers $\mathrm{u}^{*}-\mathrm{u}^{*}, \mathrm{~d}^{*} / \mathrm{d}^{*}$ and $\mathrm{u}^{*} / \mathrm{u}^{*}$ are indeterminate but indeterminacy is avoided by computation with the g- or dterms.

Clearly, $\mathrm{d}^{*}$ and $\mathrm{u}^{*}$ are the well defined counterparts of the ill-defined infinitesimal and infinity of calculus. Unlike the real numbers which are not linearly ordered by " $<$ " the new real numbers, particularly, the decimals $\mathbf{D}$, are by the lexicographic ordering " $<$ " defined as follows: two elements of $\mathbf{D}$ are equal if their corresponding integral and decimal parts are equal. Let

$$
\text { N. } a_{1} a_{2} \cdots M \cdot b_{1} b_{2} \cdots \in \mathbf{D} \text {. }
$$

Then,

$$
\begin{aligned}
& \text { N. } a_{1} a_{2} \cdots<M . b_{1} b_{2} \text { if } N<M \text { or } \\
& \text { if } N=M, a_{1}<b_{1} ; \text { if } a_{1}=b_{1}, a_{2}<b_{2} ; \cdots,
\end{aligned}
$$

and, if $\mathrm{x}$ is any decimal we have, $0<\mathrm{d}^{*}<\mathrm{x}<\mathrm{u}^{*}$.

The trichotomy axiom follows from the lexicographic ordering of the decimals.

\section{The Decimal Integers}

To find out more about the structure of $\mathbf{R}^{*}$ we show the isomorphism between the integers and decimal integers, i.e., integers of the form,

$$
\text { N.99 } \cdots, \mathrm{N}=0,1, \cdots \text {, }
$$

but we first note that $1+0.99 \cdots$ is not defined in $\mathbf{R}$ since $0.99 \cdots$ is nonterminating but we can write $0.99 \cdots=1-\mathrm{d}^{*}$ so that $1+0.99 \cdots=1+1-\mathrm{d}^{*}=2-\mathrm{d}^{*}=1.99 \cdots$; we now define $1+0.99 \cdots=1.99 \cdots$ or, in general,

$$
\mathrm{N}-\mathrm{d}^{*}=(\mathrm{N}-1) .99 \cdots, \mathrm{N}=1,2, \cdots
$$

The pairs

$$
(\mathrm{N},(\mathrm{N}-1) .99 \cdots), \mathrm{N}=1,2, \cdots,
$$

are called twin integers; their components are isomorphic.

Let $\mathrm{f}$ be the mapping $\mathrm{N} \rightarrow(\mathrm{N}-1) .99 \cdots$ and extend it to include the mapping $\mathrm{d}^{*} \rightarrow 0$; we show that $\mathrm{f}$ is an isomorphism between the integers and decimal integers.

(a)

$$
\begin{aligned}
& \mathrm{f}(\mathrm{N}+\mathrm{M}) \\
& =(\mathrm{N}+\mathrm{M}-1) \cdot 99 \cdots=\mathrm{N}+\mathrm{M}-1+0.99 \cdots \\
& =\mathrm{N}-1+\mathrm{M}-1+1.99 \cdots \\
& =\mathrm{N}-1+0.99 \cdots+\mathrm{M}-1+0.99 \cdots \\
& =(\mathrm{N}-1) \cdot 99 \cdots+(\mathrm{M}-1) \cdot 99 \cdots \\
& =\mathrm{f}(\mathrm{N})+\mathrm{f}(\mathrm{M})
\end{aligned}
$$

Next, we show that multiplication is also an isomorphism.

$$
\begin{aligned}
\text { (b) } \mathrm{f}(\mathrm{NM})= & (\mathrm{NM}-1) \cdot 999 \cdots=\mathrm{NM}-1+0.99 \cdots \\
= & \mathrm{NM}-\mathrm{N}-\mathrm{M}+1+\mathrm{N}+(-1) \\
& +\mathrm{M}+(-1)+0.99 \cdots \\
= & \mathrm{NM}-\mathrm{N}-\mathrm{M}+1+(\mathrm{N}-1) .99 \cdots \\
& +(\mathrm{M}-1) .99 \cdots+(-1)(0.99 \cdots \\
= & \mathrm{NM}-\mathrm{N}-\mathrm{M}+1+\mathrm{N}(0.99 \cdots+(-1)(0.99 \cdots)) \\
& +\mathrm{M}(0.99 \cdots+(-1)(0.99 \cdots)+0.99 \cdots \\
= & (\mathrm{N}-1)(\mathrm{M}-1)+(\mathrm{N}-1)(0.99 \cdots) \\
& +(\mathrm{M}-1)\left(0.99 \cdots+(0.99 \cdots)^{2}\right. \\
= & ((\mathrm{N}-1)+0.99 \cdots \mathrm{M}-1)+0.99 \cdots \\
= & (((\mathrm{N}-1) .99 \cdots)((\mathrm{M}-1) .99 \cdots) \\
= & (\mathrm{f}(\mathrm{N}))(\mathrm{f}(\mathrm{M})) .
\end{aligned}
$$

We have now established the isomorphism between the integers and decimal integers with respect to both operations so that both subspaces of $\mathbf{R}^{*}$ are integers in the sense of Ito, 1993 which proves that the decimal integers are integers. The kernel of this isomorphism is $\left\{d^{*}, 1\right)$ from which follows the equation,

$$
\left(d^{*}\right)^{\mathrm{n}}=\mathrm{d}^{*} \text { and }(0.99 \cdots)^{\mathrm{n}}=0.99 \cdots, \mathrm{n}=1,2, \cdots .
$$

We exhibit other properties of $0.99 \cdots$ Let $\mathrm{K}$ be an integer, M.99 $\cdots$ and N.99 $\cdots$ decimal integers. Then

$$
\begin{aligned}
& \text { (a) } \mathrm{K}+\mathrm{M} .99 \cdots=(\mathrm{K}+\mathrm{M}) .99 \cdots \text {, } \\
& \text { (b) } \mathrm{K}(\mathrm{M} .99 \ldots)=\mathrm{K}(\mathrm{M}+0.99 \cdots) \\
& =\mathrm{KM}+\mathrm{K}(0.99 \cdots) \text {, } \\
& \text { (c) } \mathrm{M} .99 \cdots+\mathrm{N} .99 \cdots=\mathrm{M}+\mathrm{N}+2(0.99 \cdots) \\
& =\mathrm{M}+\mathrm{N}+1.99 \cdots \text {, }
\end{aligned}
$$

since $(1.99 \cdots) / 2=0.99 \cdots$

$$
\begin{aligned}
(\mathrm{d}) & (\mathrm{M} .99 \cdots)(\mathrm{N} .99 \cdots) \\
& =(\mathrm{M}+0.99 \cdots)(\mathrm{N}+0.99 \cdots) \\
& =\mathrm{MN}+\mathrm{M}(0.99 \cdots)+\mathrm{N}(0.99 \cdots)+(0.99 \cdots)^{2} \\
& =\mathrm{MN}+(\mathrm{M}-1) .99 \cdots+(\mathrm{N}-1) .99 \cdots+0.99 \cdots \\
& =\mathrm{MN}+(\mathrm{M}+\mathrm{N}-2) .99 \cdots \\
& =(\mathrm{MN}+\mathrm{M}+\mathrm{N}-1) .99 \cdots,
\end{aligned}
$$

\section{Adjacent Decimals and Recurring 9s}

Two decimals are adjacent if they differ by $\mathrm{d}^{*}$. Predecessorsuccessor pairs and twin integers are adjacent; e.g., 74.5700 .. and $74.5699 \cdots$ are adjacent.

Since the decimals have the form $N . a_{1} a_{2} \cdots a_{n}, \cdots, N=0,1$, $2, \cdots$, the digits are identifiable and, in fact, countably infinite and discrete except their g-limits which form a continuum. They are the union of pairwise adjacent predecessor-successor pairs and linearly ordered by the lexicographic ordering. Moreover, since the decimals are joined together by the continuum $\mathrm{d}^{*}$ as pairwise adjacent pairs and at their tails, $\mathbf{R}^{*}$ is a continuum with the decimals its discrete countably infinite subspace. A 
decimal is called recurring 9 if its tail decimal digits are all equal to 9, e.g., $4.3299 \cdots$ and $299.99 \cdots$, if its decimal digits are all equal to 9 it is called decimal integer.

The recurring $9 \mathrm{~s}$ have interesting properties, e.g., the difference between the integer $\mathrm{N}$ and the recurring 9, $(\mathrm{N}-1) .99 \cdots$, is $\mathrm{d}^{*}$. Such pairs are called adjacent because there is no decimal between them and they differ by $\mathrm{d}^{*}$. The average between them is the predecessor, e.g., the average between 1 and $0.99 \cdots$ is $0.99 \cdots$ Moreover, the g-limit of the iterated or successive averages between a fixed decimal and another decimal of the same integral part is the predecessor of the former.

Since adjacent decimals differ by $\mathrm{d}^{*}$ and there is no decimal between them, $\mathrm{d}^{*}$ cannot be split from a decimal.

The counterexample to the trichotomy axiom shows that an irrational real number cannot be expressed as limit of sequence of rationals. Thus, irrational is ill-defined in both $\mathbf{R}$ and $\mathbf{R}^{*}$.

The g-sequence of a nonterminating decimal gets directly to its g-limit, digit by digit. Moreover, a nonterminating decimal is the infinite series of its digits:

$$
\begin{aligned}
& \text { N. } \mathrm{a}_{1} \mathrm{a}_{2} \cdots \mathrm{a}_{\mathrm{n}} \cdots=\mathrm{N}+. \mathrm{a}_{1}+.0 \mathrm{a}_{2}+\cdots+.00 \cdots 0 \mathrm{a}_{\mathrm{n}}+\cdots ; \\
& 0.99 \cdots=0.9+0.09+\cdots
\end{aligned}
$$

\section{R* and Its Subspaces}

The following applies to subspaces of $\mathbf{R}^{*}$ including the real numbers (terminating decimals).

Theorem. In the lexicographic ordering $\mathbf{R}^{*}$ consists of adjacent predecessor-successor pairs (each joined by $\mathrm{d}^{*}$ ); hence, the g-closure $\mathbf{R}^{*}$ of $\mathbf{R}$ is a continuum (Escultura, 2009b).

Proof. For each $\mathrm{N}, \mathrm{N}=0,1, \cdots$, consider the set of decimals with integral part N. Take any decimal there, say, N. $\mathrm{a}_{1} \mathrm{a}_{2} \cdots$, and another in it. Without loss of generality, let N. $\mathrm{a}_{1} \mathrm{a}_{2} \cdots$ be fixed and let it be the larger decimal. We take the average of the nth g-terms of $\mathrm{N}_{\mathrm{a}} \mathrm{a}_{2} \cdots$ and the second decimal; then take the average of the nth g-terms of this average and $\mathrm{N}_{\mathrm{a}} \mathrm{a}_{2} \ldots$; continue indefinitely. We obtain the d-sequence with nth d-term, $(0.5)^{-\mathrm{n}} \mathrm{a}_{1} \mathrm{a}_{2} \cdots \mathrm{a}_{\mathrm{n}+\mathrm{k}}$, which is a d-sequence of $\mathrm{d}^{*}$. Therefore, the g-limit of this sequence of averages is the predecessor of $\mathrm{N}_{1} \mathrm{a}_{1} \mathrm{a}_{2} \cdots$ and we have proved that this g-limit and $\mathrm{N}_{\mathrm{a}} \mathrm{a}_{2} \cdots$ are predecessor-successor pair, differ by $\mathrm{d}^{*}$ and form a continuum. Since the choice of $\mathrm{N}_{\mathrm{a}} \mathrm{a}_{1} \mathrm{a}_{2} \cdots$ is arbitrary then by taking the union of these predecessor-successor pairs of decimals in $\mathbf{R}^{*}$ (each joined by the continuum $\mathrm{d}^{*}$ ) for all integral parts $\mathrm{N}, \mathrm{N}=0,1, \cdots$, we establish that $\mathbf{R}^{*}$ is a continuum.

However, the decimals form countably infinite discrete subspace of $\mathbf{R}^{*}$ since there is a scheme for labeling them by integers.

Corollary. $\mathbf{R}^{*}$ is non-Archimedean and non-Hausdorff in both the standard and g-norms but the subspace of decimals are countably infinite and discrete, Archimedean and Hausdorff.

The following theorem is true in the subspace of decimals. Therefore, we do not bring in $\mathrm{d}^{*}$ in the proof so that this is really a theorem about the decimals with the standard norm which is not true in the g-norm because the decimals merge into a continuum at their tail digits and cannot be separated.

Theorem. Every decimal is isolated from the rest of the decimals (Escultura, 2003).

Proof. Let $\mathrm{p} \in \mathrm{R}$ be any irrational number and $\left\{\mathrm{q}_{\mathrm{n}}\right\}$ a sequence of rationals converging to $p$ from the left. Let $d_{n}$ be the distance from $q_{n}$ to $p$ and take an open ball of radius $d_{n} / 10^{n}$, with center at $\mathrm{q}_{\mathrm{n}}$. Note that $\mathrm{q}_{\mathrm{n}}$ tends to $\mathrm{p}$ but distinct from it for any n. Take an open ball of radius $d_{n} / 10^{n}$, centered at $p$ and take the union of open balls, centered at $\mathrm{q}_{\mathrm{n}}$, as $\mathrm{n} \rightarrow \infty$ and call it $\mathrm{U}$. If $r$ is any real, rational or irrational, to the left of $p$, then $r$ is separated from $\mathrm{p}$ by two disjoint open balls, one in $U$ and the other in its complement, center at $p$. If $p$ is rational, then we take $\left\{\mathrm{q}_{\mathrm{n}}\right\}$ as a sequence of irrationals that tend to $\mathrm{p}$, which is allowed by the Axiom of Choice (Escultura, 2002, 2003). The same result will hold for any $\mathrm{r}$ distinct from and to the right of $\mathrm{p}$.

Theorem. The terminating and nonterminating decimals are separated, i.e., not dense in their union (first indication of discreteness).

Proof. Let $p \in \mathbf{R}$ (the real numbers including the ambiguous irrationals with the standard norm) be an irrational number and let $\mathrm{q}_{\mathrm{n}}, \mathrm{n}=1,2, \cdots$, be a sequence of rationals towards and left of $p$, i.e., $n>m$ implies $q_{n}>q_{m}$; let $d_{n}$ be the distance from $q_{n}$ to $\mathrm{p}$ and take an open ball of radius $\mathrm{d}_{\mathrm{n}} / 10^{\mathrm{n}}$, center at $\mathrm{q}_{\mathrm{n}}$. Note that $\mathrm{q}_{\mathrm{n}}$ tends to $\mathrm{p}$ but distinct from it for any $\mathrm{n}$. Let $\mathrm{U}=\cup \mathrm{U}_{n}$, as $\mathrm{n} \rightarrow \infty$, then $\mathrm{U}$ is open and if $\mathrm{q}$ is any real number, rational or irrational, to the left of $\mathrm{p}$ then $\mathrm{q}$ is separated from $\mathrm{p}$ by disjoint open balls, one in $\mathrm{U}$, center at q, and the other in the complement of $U$. Since the rationals are countable the union of open sets $\mathrm{U}, \mathrm{q}_{\mathrm{n}}, \mathrm{q}^{\mathrm{n}}$ rational, $\mathrm{n}=1,2, \cdots$, and the irrational $\mathrm{p}$ is separated from the rationals (Escultura, 2002, 2003).

We use the same argument if $\mathrm{p}$ were rational and since the real number system has countable basis we take $\mathrm{q}_{\mathrm{n}}$ an irrational number, for each $n$, at center of open ball of radius $d_{n} / 10^{n}$. Take $U$ to be the union of such open balls. Using the same argument a real number in $\mathrm{U}$, rational or irrational, is separated by disjoint open balls from $\mathrm{p}$. The proof is standard in the real line (Escultura, 2003).

Thus, every decimal is separated from the rest of $\mathbf{R}$, the terminating decimals from the nonterminating decimals and from each other (not true in $\mathbf{R}^{*}$ ). The following theorem has, again, a standard proof in $\mathbf{R}$ which is also true in $\mathbf{R}^{*}$.

Theorem. The largest and smallest elements of the open interval $(0,1)$ are $0.99 \cdots$ and $d^{*}$, respectively (Escultura, 2002).

Proof. Let $C_{n}$ be the nth term of the g-sequence of $0.99 \ldots$ For each $n$, let $I_{n}$ be an open segment (segment that excludes its endpoints) of radius $10^{-2 n}$ centered at $C_{n}$. Since $C_{n}$ lies in $I_{n}$ for each $n, C_{n}$ lies in $(0,1)$ as $n$ increases indefinitely. Therefore, the decimal $0.99 \cdots$ lies in the open interval $(0,1)$ and never reaches 1 . To prove that $0.99 \cdots$ is the largest decimal in the open interval $(0,1)$ let $\mathrm{x}$ be any point in $(0,1)$. Then $x$ is less than 1 . Since $C_{n}$ is steadily increasing $n$ can be chosen large enough so that $\mathrm{x}$ is less than $\mathrm{C}_{\mathrm{n}}$ and this is so for all subsequent values of $\mathrm{n}$. Therefore, $\mathrm{x}$ is less than $0.99 \cdots$ and since $\mathrm{x}$ is any decimal in the open interval $(0,1)$ then $0.99 \cdots$ is, indeed, the largest decimal in the interval and is itself less than 1.

To prove that $1-0.99 \cdots$ is the smallest element of $\mathbf{R}$, we note that the g-sequence of $1-0.99 \cdots$ in (14) is steadily decreasing. Let $K_{n}$ be the $n$th term of its g-sequence. For each $n$, let $B_{n}$ be an open interval with radius $10^{-2 n}$ centered at $k_{n}$. Then $\mathrm{K}_{\mathrm{n}}$ lies in $\mathrm{B}_{\mathrm{n}}$ for each $\mathrm{n}$ and all the $\mathrm{B}_{\mathrm{n}} \mathrm{s}$ lie in the open set in $(0,1)$. If $y$ is any point of $(0,1)$, then $y$ is greater than 0 and since the generating sequence $1-0.99 \cdots$ is steadily decreasing $n$ can be chosen large enough such that $\mathrm{y}$ is greater than $\mathrm{K}_{\mathrm{n}}$ and this is so for all subsequent values of $\mathrm{n}$. Therefore, $\mathrm{y}$ is greater than $=1-0.99 \cdots$ and since the choice of $\mathrm{y}$ is arbitrary, $1-$ $0.9 \cdots$ is the smallest number in the open interval $(0,1)$; at the same time $1-0.99 \cdots$ is greater than $0 . \square$

This theorem is true in $\mathbf{R}$ with $1-0.99 \cdots$ replaced by $\mathrm{d}^{*}$ 
and follows from properties of terminating decimals.

Theorem. An even number greater than 2 is the sum of two primes (formerly called Goldbach's conjecture (Davies \& Hersch, 1981).

Like Fermat's equation (FLT) (Escultura, 1998), this conjecture in the real number system is indeterminate, the reason it could not be solved. We first note that an integer is prime if it leaves a positive remainder when divided by an integer. We retain this definition in $\mathbf{R}^{*}$.

Proof. The conjecture is obvious for $n<10$. Let $n$ be even greater than $10, \mathrm{p}, \mathrm{q}$ integers and $\mathrm{p}$ prime. If $\mathrm{q}$ is prime the theorem is proved; otherwise, it is divisible by an integer other than 1 and $\mathrm{q}$. Since $\mathrm{d}^{*}$ cannot be separated from any decimal, dividing $\mathrm{q}$ by an integer other than 1 and $\mathrm{q}$ leaves the remainder $\mathrm{d}^{*}>0$. Therefore, $\mathrm{q}$ is prime. $\square$

\section{Other Important Results}

1) Every convergent sequence has g-subsequence that defines an adjacent decimal to its limit in the standard norm. If the decimal is terminating it is the limit itself.

2) It follows from (1) that the limit of a sequence of terminating decimals can be found by evaluating the g-limit of its g-subsequence which is adjacent to it. We use this as alternative way of computing the limit of ordinary sequence.

3) In Horgan, 1993, several counterexamples to the generalized Jourdan curve theorem for n-sphere are shown where a continuous curve has points in both the interior and exterior of the $n$-sphere, $n=2,3, \cdots$, without crossing the $n$-sphere. The explanation: the functions cross the n-sphere through dark numbers.

5) Given two decimals and their g-sequences and respective nth g-terms $A_{n}, B_{n}$ we define the nth g-distance as the g-norm $\left|A_{n}-B_{n}\right|$ of the difference between their nth g-terms. Their g-distance is the g-lim $\left|A_{n}-B_{n}\right|$, as $n \rightarrow \infty$, which is adjacent to the standard norm of the difference (9). Advantage: the g-distance is the g-norm of their decimal difference; the difference between nonterminating decimals cannot be evaluated otherwise. Moreover, this notion of distance can be extended to $\mathrm{n}$-space, $\mathrm{n}=2,3, \cdots$, and the distance between two points can be evaluated digit by digit in terms of their components without the need for evaluating roots. In fact, any computation in the g-norm yields the results directly, digit by digit, without intermediate computation.

\section{The Counterexamples to FLT}

Fermat's last theorem states: Given integer $n>2$, Fermat's equation,

$$
x^{n}+y^{n}=z^{n}
$$

has no solution in nonzero integers $\mathrm{x}, \mathrm{y}, \mathrm{z}$ (Escultura, 1998).

This "theorem" is actually a conjecture by Fermat because he did not provide the proof.

Fermat's Equation (29) is, of course, ambiguous in the real number system being inconsistent and, in particular, the integers are not even defined there. This was, in fact, the catalyst for the development of the consistent new real number system which resolves this conjecture.

We consider Fermat's equation in place of Fermat's last theorem (FLT) so that its solution is a counterexample to FLT that proves it false. We first summarize the properties of the digit or basic integer 9 .
1) A string of $9 \mathrm{~s}$ differs from the nearest power of 10 by 1 , e.g., $10^{100}-99 \ldots 9=1$.

2) If $\mathrm{N}$ is an integer, then $(0.99 \cdots)^{\mathrm{N}}=0.99 \cdots$ and, naturally, the two sides of this equation have equivalent $\mathrm{g}$-sequences. Therefore, for any integer $\mathrm{N},((0.99 \cdots) 10)^{\mathrm{N}}=(9.99 \cdots) 10^{\mathrm{N}}$.

3) $\left(d^{*}\right)^{N}=d^{*} ;((0.99 \cdots,) 10)^{N}+d^{*}=10^{N}, N=1,2, \cdots$

Then the exact solutions of Fermat's equation are the triples $(\mathrm{x}, \mathrm{y}, \mathrm{z})=\left((0.99 \cdots) 10^{\mathrm{T}}, \mathrm{d}^{*}, 10^{\mathrm{T}}\right), \mathrm{T}=1,2, \cdots$, that clearly satisfy Fermat's equation,

$$
\mathrm{x}^{\mathrm{n}}+\mathrm{y}^{\mathrm{n}}=\mathrm{z}^{\mathrm{n}}
$$

for $\mathrm{n}=\mathrm{NT}>2$. Moreover, for $\mathrm{k}=2,3, \cdots$, the triples $(\mathrm{kx}$, ky, kz) also satisfy Fermat's equation. They are the countably infinite counterexamples to FLT that prove the conjecture false (Escultura, 1998). One counterexample is, of course, sufficient to disprove a conjecture but we got more-a countable infinity of them.

\section{The Complex Vector Plane C}

Due to contradictions in the concept $i$ we replace it by a vector operator $\mathbf{j}$ and build the consistent complex vector plane (see Appendix to Escultura, in press).

\section{The Number $\mathbf{j}$ as Operator on Plane Vectors}

We introduce the operator $\mathbf{j}$ in place of $\mathbf{i}$ and define $\mathbf{j}$ as left-right operator on or mapping of a plane vector by positive or counterclockwise rotation about the origin through $\pi / 2$. Then we generate the coordinate axes by applying $\mathbf{j}$ on the unit vector $\mathbf{1}$ along the $\mathrm{x}$-axis, i.e., $\mathbf{j}(\mathbf{1})=\mathbf{j}$, the unit vector along the $\mathrm{y}$-axis or $\mathbf{j} y$, then on $\mathbf{j}$ to obtain $\mathbf{j} \mathbf{j}=-\mathbf{1}$, the unit vector along the negative $\mathrm{x}$-axis or $-\mathrm{x}$, then on $-\mathbf{1}$, to obtain $\mathbf{j}(-\mathbf{1})=-\mathbf{j}$, the unit vector along the negative $\mathbf{j y} y$-axis or $-\mathbf{j} y$, and then on $-\mathbf{j}$ to obtain $-\mathbf{j j}=\mathbf{1}$, back to the unit vector along the $x$-axis or $x$. The cyclic values of the composites of $\mathbf{j}$ are:

$$
\mathrm{j}, \mathrm{j}(\mathrm{j})=\mathrm{j}^{2}=-\mathbf{1}, \mathrm{j}\left(\mathrm{j}^{2}\right)=\mathrm{j}^{3}=\mathrm{j}(\mathrm{i}), \mathrm{j}\left(\mathrm{j}^{3}\right)=\mathrm{j}^{4}=\mathbf{1} \text {. }
$$

We define $-\mathbf{j}$ as inverse operator of $\mathbf{j}$, i.e., clockwise rotation of the unit vector 1 along the $x$-axis by $\pi / 2$. Note: $-\mathbf{j}(\mathbf{1})=\mathbf{j}(-\mathbf{1})$. Applying composite mappings on the unit vector 1 along the $x$-axis successively, we have the four cyclic images of 1 in (31) under the composites of the operator $\mathbf{j}$ along the $\mathbf{j y}-,-\mathbf{x}-,-\mathbf{j y}$ and $\mathrm{x}$-axes, respectively. For $\mathrm{n}>4$, the cycle is repeated and we define $\mathbf{j}^{\mathbf{n}}=\mathbf{j}\left(\mathbf{j}^{\mathrm{n}-1}\right), \mathrm{n}=1,2, \cdots$, where we define $\mathbf{j}^{0}=\mathbf{1}$.

\section{Scalar and Vector Operations}

For completeness, we introduce scalar multiplication. If $\alpha$ is an element of $\mathbf{R}^{*}$, called scalar, $\alpha \mathbf{j}=\mathbf{j} \alpha$, is a vector of modulus $\alpha$ along the $\mathbf{j y}$-axis so that $\alpha$ commutes with $\mathbf{j}$, the unit complex vector operator. If $b$ is another scalar,

$$
(\alpha \beta) \mathbf{j}=\mathbf{j}(\alpha \beta)=(\mathbf{j} \alpha) \beta=\beta(\mathbf{j} \alpha)=(\beta \alpha) \mathbf{j},
$$

which follows from $\mathbf{j}$ being left-right operator and the commutativity of multiplication in $\mathbf{R}^{*}$. From commutativity and associativity of multiplication we have, for $\alpha, \beta, \gamma \in \mathbf{R}^{*}$,

$$
\begin{aligned}
(\alpha \beta \gamma) \mathbf{j} & =\alpha(\beta \gamma) \mathbf{j}=(\alpha \beta)(\gamma \mathbf{j})=(\beta \alpha) \gamma \mathbf{j} \\
& =(\gamma \beta \alpha) \mathbf{j}=\mathbf{j}(\gamma \beta \alpha) .
\end{aligned}
$$

Also, from distributivity of multiplication in $\mathbf{R}^{*}$ with respect 


\section{E. E. ESCULTURA}

to addition we have,

$$
\alpha(\beta+\gamma) \mathbf{j}=(\alpha \beta+\alpha \gamma) \mathbf{j}=(\alpha \beta) \mathbf{j}+(\alpha \gamma) \mathbf{j} .
$$

Thus, we have retrieved the basic properties of the complex plane.

Every vector in the complex vector plane has new real and complex components; conversely, a vector is the vector sum of its new real and complex components. Thus, a vector $\mathbf{z}$ in it has standard form,

$$
\mathbf{z}=\alpha+\beta \mathbf{j} \text { or } \mathbf{z}=(\mathrm{a}, \beta \mathbf{j}), \alpha, \beta \in \mathbf{R}^{*}
$$

The arithmetic of the complex plane holds provided that whenever $\mathbf{1}$ appears as a factor we interpret it as a unitary vector operator so that $1 \alpha=\alpha$, the vector of modulus $\alpha$ along the $\mathrm{x}$-axis. Thus, we retain in the complex vector plane the vector algebra of the complex plane, the latter embedded in the former isomorphically. All concepts of the complex plane except $i$ carry over to the complex vector plane, e.g., the norm or modulus of the complex vector $\mathbf{z}=\alpha+\beta \mathbf{j}$, denoted by $|\mathbf{z}|$, is given by,

$$
|\mathbf{z}|=\left(\alpha^{2}+\beta^{2}\right)^{1 / 2},
$$

the square root of the product of $\mathbf{z}$ and its conjugate, $\alpha-\beta \mathbf{j}$. The dot product of vectors $\mathbf{u}$ and $\mathbf{v}$ is given by

$$
\begin{aligned}
& \mathbf{u} \cdot \mathbf{v}=|\mathbf{u}||\mathbf{v}| \cos \theta \text { if } \mathbf{u} \neq \mathbf{0}, \mathbf{v} \neq \mathbf{0} \\
& \mathbf{u} \cdot \mathbf{v}=0 \text { if } \mathbf{u}=\mathbf{0} \text { or } \mathbf{v}=\mathbf{0},
\end{aligned}
$$

where $\theta$ is a new real number.

We use an arrow with initial and terminal points A, B to represent a vector with direction from A to B. Two parallel vectors with the same norm and direction are equivalent. Therefore, a vector can be translated so that its initial point lies in the origin. This is called standard vector and has standard representation of the form (35).

The vector additive and multiplicative identities are $\mathbf{0}$ and $\mathbf{1}$, respectively, where the norm of latter called the unit vector coincides with its real component 1 . The scalars (new reals) are commutative and associative with respect to the additive and multiplicative operations in $\mathbf{R}^{*}$ and as scalar operations on plane vectors. With the complex vector arithmetic now defined we have verified that the operator $\mathbf{j}$ applies to any vector in the complex vector plane. Applying $\mathbf{j}$ on the vector $\mathbf{z}$ of (35), we have,

$$
\mathbf{j}(\mathbf{z})=\mathbf{j}(\alpha+\mathbf{j} \beta)=-\beta+\mathbf{j} \alpha,
$$

a positive rotation of vector $\mathbf{z}$ by $\pi / 2$.

\section{The Operator $\mathbf{h}_{\theta}$}

We introduce a more general complex left-right operator on the complex vector plane appropriate for analytical work:

$$
\mathbf{h}_{\theta}(\mathrm{a}+\mathbf{j b})=\mathrm{r}(\cos \theta+\mathbf{j} \sin \theta),
$$

a rotation of the unit vector 1 around the terminal point of the vector $\alpha+\mathbf{j} \beta$ by $\theta$, an element of $\mathbf{R}^{*}$. We can represent a vector $\mathbf{z}$ in the complex vector plane as,

$$
\left.\mathbf{z}=\mathbf{h}_{\theta} \alpha+\mathbf{j} \beta\right)=r(\cos \theta+\mathbf{j} \sin \theta),
$$

where $r$ is the modulus of $\mathbf{z}$ and $\theta$ its argument. This is the well defined counterpart of $\mathrm{e}^{\mathrm{i} \theta}$. If we vary $\alpha$ and $\beta$ along $\mathbf{R}^{*}$ and $\theta$ in $[0,2 \pi]$ the terminal point of $\mathbf{z}$ covers the entire complex vector plane. Geometrically, $r$ varies in $[0, \infty)$ and rotates around the origin from 0 to $2 \pi$ as the unit circle with center at the terminal point of $\alpha+\mathbf{j} \beta$ rotates from $\theta=0$ to $\theta=2 \pi$. Then a point $z_{0}$ is given by

$$
\mathbf{z}_{0}=\mathbf{h}_{\theta}\left(\alpha_{0}+\mathbf{j} \beta_{0}\right)=\mathrm{r}_{0}\left(\cos \theta_{0}+\mathbf{j} \sin \theta_{0}\right), \theta_{0} \in[0,2 \pi] .
$$

If $r_{0}=1$, (41) reduces to the equation of the unit circle with center at the origin. This operator applied on a vector along the $\mathrm{x}$-axis rotates it by $\theta$, reducing to operator $\mathbf{j}$ when $\theta=\pi / 2$.

In the solution of the gravitational n-body problem (4), the operator that generates the spiral covering of a vortex is a variant of $\mathbf{h}_{\theta}$ and has the form,

$$
\mathrm{z}=\mathrm{ae}^{\lambda \mathrm{t}} \eta(\mathrm{t})
$$

where $\eta$ is given by the expression,

$$
\mathbf{h}_{\lambda}(\mathrm{t})=\cos \lambda+\mathrm{je}^{-\mathrm{t}} \sin \lambda,
$$

depending on the specific cases and phases of the evolving boundary conditions of the problem; here $\lambda$ is constant of integration in the solution of the constraint equation of the associated optimal control formulation of this problem (20).

Suppose vector $\mathbf{z}$ has initial and terminal points $(\alpha, \mathbf{j} \beta)$ and $(\gamma, \mathbf{j} \zeta)$, respectively. Then,

$$
\mathbf{z}=\mathbf{z}_{1}-\mathbf{z}_{2}=(\alpha, \mathbf{j} \beta)-(\gamma, \mathbf{j} \zeta)
$$

Therefore,

$$
\begin{aligned}
\mathbf{j}(\mathbf{z}) & =\mathbf{j}\left(\mathbf{z}_{1}-\mathbf{z}_{2}\right)=\mathbf{j}((\alpha+\mathbf{j} \gamma \beta)-(\gamma+\mathbf{j} \zeta)) \\
& =\mathbf{j}(\alpha-\gamma)+(\zeta-\beta)=(\zeta-\beta)+\mathbf{j}(\alpha-\gamma),
\end{aligned}
$$

is counterclockwise rotation of $\mathbf{z}$ by $\pi / 2$. A polygon of $n$ edges $\mathbf{e}_{1}, \cdots, \mathbf{e}_{\mathrm{n}}$ may be represented as the vector sum $\mathbf{e}_{1}+\cdots+\mathbf{e}_{\mathrm{n}}$ or its resultant $\mathbf{r}$. Then $\mathbf{j}\left(\mathbf{e}_{1}+\cdots+\mathbf{e}_{n}\right)=\mathbf{j}(\mathbf{r})$ is a counterclockwise rotation of the polygon by $\pi / 2$.

The operator $\mathbf{j}$ is an automorphism of the complex vector plane. Its additive inverse $-\mathbf{j}$ is its clockwise rotation about the origin by $\pi / 2$.

There is, however, a new vector operation in the complex vector plane that has no counterpart in other vector spaces: the product of two vectors. Let $\mathbf{u}=\alpha+\mathbf{j} \beta, \mathbf{v}=\gamma+\mathbf{j} \zeta$, their product is given by

$$
\mathbf{u v}=(\alpha+\mathbf{j} \beta)(\gamma+\mathbf{j} \zeta)=(\alpha \gamma-\beta \zeta)+\mathbf{j}(\alpha \zeta+\beta \gamma)
$$

that, restricted to the complex vector plane, reduces to standard complex vector multiplication with $\mathbf{j}$ replaced by $i$. This is a particularity of the complex vector plane not shared by other vectors spaces. Consider the vectors,

$$
\mathbf{z}_{1}=(\alpha+\mathbf{j} \beta), \quad \mathbf{z}_{2}=(\gamma+\mathbf{j} \zeta)
$$

then,

$$
\begin{aligned}
\mathbf{z}_{1} \mathbf{z}_{2} & =\mathbf{h}_{\theta}(\alpha+\mathbf{j} \beta) \mathbf{h}_{\varphi}(\gamma+\mathbf{j} \zeta) \\
& =\mathrm{r}_{1} \mathrm{r}_{2}(\cos (\theta+\varphi)+\mathbf{j} \sin (\theta+\varphi)),
\end{aligned}
$$

where $r_{1}, r_{2}$ are the respective moduli of the vectors of $\mathbf{z}_{1}, \mathbf{z}_{2}$ and $\theta, \varphi$ their arguments. Note that this product of complex vectors is distinct from both the dot and vector products in a vector space. It is an extension of multiplication of complex numbers. Since the product of two complex vectors is a complex vector the product vector can be extended to any number of factors.

The additive inverse of a complex vector is obvious. For the 
multiplicative inverse we reduce its reciprocal to standard form. For instance, if $\mathbf{z}=\alpha+\mathbf{j} \beta$ then its multiplicative inverse $\mathbf{z}^{-1}$ is given by

$$
\begin{aligned}
\mathbf{z}^{-1} & =1 /(\alpha+\mathbf{j} \beta)=(\alpha-\mathbf{j} \beta) /\left(\mathrm{a}^{2}+\mathrm{b}^{2}\right) \\
& =\mathbf{h}\left(\mathbf{z}^{-1}\right)=\mathbf{j}(1 / \mathrm{r})(\cos \theta-\mathbf{j} \sin \theta),
\end{aligned}
$$

where $1 / r=\bmod \left(\mathbf{z}^{-1}\right)$ and $\theta=\arg \left(\mathbf{z}^{-1}\right)$. Then division of complex vector by another reduces to its multiplication by the inverse of the other. In general, if $\mathbf{z}_{1}=r_{1}(\cos \theta+\mathbf{j} \sin \theta)$,

$\mathbf{z}_{2}=r_{2}(\cos \varphi+\mathbf{j} \sin \varphi)$, then $\mathbf{z}_{1} / \mathbf{z}_{2}=\left(r_{1} / r_{2}\right)(\cos \theta-\sin \varphi)$.

Note that the operator $\mathbf{h}_{\theta}$ is really equivalent to the old notation $\mathrm{e}^{\mathrm{j} \theta}$ and the latter may be used in place of $\mathbf{h}_{\theta}$.

The operator $\mathbf{j}$ played a crucial role in solving the gravitational n-body problem (Escultura, 1997) by generating the spiral covering of the underlying vortex by the gravitational flux streamlines as solutions of the conjugate equations obtained by the integrated Pontrjagin maximum principle from its optimal control formulation (Young, Lectures, 1969). The $\mathrm{n}$ bodies and their rotating trajectories were obtained along specific spiral streamlines by the fractal-reverse-fractal algorithm (Escultura, 2011) using a body at the core of the vortex as fractal generator. Elliptical orbit in the underlying spinning vortex is attained when the gravitational flux pressure balances the centrifugal force, its ellipticity being due to radial fluctuation of this balance by virtue of the oscillation universality principle, another expression of perfect balance being unstable which accounts for the fact that orbits of cosmological bodies are elliptical (Escultura, 2011).

This provides the right setting for complex vector analysis on the complex vector plane as extension of $\mathbf{R}^{*}$.

\section{Concluding Remark}

We conclude this expository paper with recommendations on the distribution of the content of creative mathematics education into the various levels of the educational system from the primary years through graduate school. The transition to this new content of mathematics education will be quite extended as Discrete Calculus and Computation and the Complex Vector Plane are being developed and appropriate textbooks being written.

1) During the primary years the following will be introduced: the addition and multiplication tables to build the integers on 1 and 0 as place value Hindu Arabic numerals (scientific notation); prime and composite numbers; well defined fraction as well defined quotient (i.e., the divisor has no prime factor other than 2 or 5); addition of fractions; well defined difference and quotient (i.e., the divisor has no prime factor other than 2 or 5) of integers and fractions; integers with integral exponent and their addition, multiplication and well defined subtraction and division and practical applications.

2) The $5^{\text {th }}$ and $6^{\text {th }}$ years will cover terminating decimals and related concepts, their defined sums and quotients and conversions between defined fractions (terminating decimals). Omission of the ambiguous concept irrational will provide considerable simplification of mathematics so that the new real number system will become the disjoint union of the rationals (terminating decimals) and nonterminating decimals.

3) The $7^{\text {th }}$ through $8^{\text {th }}$ years will cover the additive and multiplicative operations and their inverses (whenever defined) not formally as part of an axiomatic system but based on practical experience and examples. There will be abundant practical applications to enhance formation of appropriate concepts. Signed numbers and the rules of signs will also be introduced here; so will addition, subtraction, multiplication and well defined quotients of signed numbers.

4) During the $9^{\text {th }}$ through $12^{\text {th }}$ years the well defined real number system consisting of terminating decimals will be introduced as a mathematical space or system based on axioms $1-3$. The role and nature of the axioms in mathematics and in constructing a mathematical space will be discussed and given emphasis. The same should be done with respect to the complex vector plane where the axioms are: 1) the existence of the unit vector $\mathbf{1}$ and 2) vector rotation. Vector addition, translation and dot product as well as inversion are introduced here. At all times, both systems will be related to experience. Fractions will be revisited as quotients where the divisors do not contain prime factors other than 2 or 5 . Then the student will have the unique experience of extending division to cases where the quotient is ill-defined, e.g., when the divisor has a prime factor other than 2 or 5 . In fact, this would be an excellent opportunity to show how a nonterminating decimal arises and how to define and compute with them (Escultura, 2009b) by giving examples. At this point the student will be introduced to some special nonterminating decimals important to science such as $\pi$ and the exponential base $e$ how they arise in mathematics.

5) The college curriculum will start with the integrated college algebra-circular functions based on the new real number system built on its axioms. The complex number system chapter of the conventional college course will be replaced by the complex vector plane. The nth root of a number will be evaluated as illustrated in Escultura, 2009b. The new real numbers, subheading Introduction to discrete computation, which can be done by the calculator. This is the same algorithm for finding roots upon which the calculator is based. Then numbers with rational exponents, i.e., fractional exponents whose denominators do not have prime factors other than 2 or 5 , can be defined and evaluated or approximated. As in the case of nonterminating decimals, numbers with non-rational exponents can be approximated by numbers with rational exponents and computed with the scientific calculator. With the methodology we have introduced here, we can figure out a suitable way to deal with all extensions of the new real number system including $\mathbf{R}^{\mathrm{n}}, \mathrm{n}=$ $2,3, \cdots$ as well as extensions of the complex vector plane. During the transition phase, the mathematics courses beyond the integrated college algebra-circular functions course built on the new real number system and complex vector plane will be taught in the traditional way while appropriate textbooks are being written. They include the calculus series, differential equations, real and complex analyses and probability theory. Topology and abstract algebra will be taught the usual way unless experts in these fields carry out similar critique-rectification. During this period the resolution of famous problems or conjectures of mathematics such as FLT will be included as part of the curriculum.

6) In the graduate school formal courses will be optional depending on what the advisor feels beneficial to the student. The advisor will serve as a role model, his publications to be studied thoroughly by his advisees. The main activity will be research, seminars, independent study, paper presentation at international mathematical conferences and publication in peer reviewed mathematical journals. Three such publications to be consoli- 


\section{E. E. ESCULTURA}

dated into a thesis should be the minimum requirement for a Ph.D.

What is being proposed is really an overhaul of both mathematics and mathematics education starting with the real and complex number systems to rid them of inadequacy and other defects. This is based on the observation that defective fields have long standing unsolved problems and research and publications there are scanty. This observation applies to number theory, real analysis, complex analysis and the theory of relativity. In the case of natural science a sure indication of its defect or inadequacy, aside from scanty research, is failure to yield a technology that works. Moreover, errors in or inadequacy of mathematics may have disastrous consequences in applications as the disastrous final flight of the Columbia Space Shuttle showed. The culprit: inadequate theory (Escultura, 2007).

Finally, we remark that the main new methodology applied here is qualitative mathematics, introduced and the main contribution of Escultura, 1970; it was applied to physics for the first time to solve the gravitational n-body problem (Escultura, 1997).

\section{REFERENCES}

Davies, P. J., \& Hersch, R. (1981). Chapter 3: Famous problems. In The Mathematical Experience (pp. 207-316). Boston: Birkhäuser.

Escultura, E. E. (1970). The trajectories, reachable set, minimal levels and chain of trajectories of a control system, Ph.D. Thesis, Madison: University of Wisconsin.

Escultura, E. E. (1997). The solution of the gravitational n-body problem. Journal of Nonlinear Analysis, A-Series: Theory, Methods and Applications, 30, 5021-5032.

Escultura, E. E. (1998). Exact solutions of Fermat's equation (A definitive resolution of Fermat's last theorem). Journal of Nonlinear Studies, 5, 227-254.

Escultura, E. E. (2002). The mathematics of the new physics. Journal of Applied Mathematics and Computations, 130, 149-169.

Escultura, E. E. (2003). The new mathematics and physics. Journal of Applied Mathematics and Computation, 138, 145-169.

Escultura, E. E. (2007). The Pillars of the new physics and some up- dates. Journal of Nonlinear Studies, 14, 241-260.

Escultura, E. E. (2008). Extending the reach of computation. Journal of Applied Mathematics Letters, 21, 1074-1081. doi:10.1016/j.aml.2007.10.027

Escultura, E. E. (2009a). The mathematics of the grand unified theory (GUT). Journal of Nonlinear Analysis, A-Series: Theory: Method and Applications, 71, e420-e431. doi:10.1016/j.na.2008.11.003

Escultura, E. E. (2009b). The new real number system and discrete computation and calculus. Journal of Neural, Parallel and Scientific Computations, 17, 59-84.

Escultura, E. E. (2009c). Qualitative model of the atom, its components and origin in the early universe. Journal of Nonlinear Analysis, BSeries: Real World Applications, 11, 29-38. doi:10.1016/j.nonrwa.2008.10.035

Escultura, E. E. (2011). Scientific natural philosophy. Chapter 3: The grand unified theory. Bentham Ebooks, 60-107. http://www.benthamscience.com/ebooks/9781608051786/index.htm

Escultura, E. E. (2011). Scientific natural philosophy. Chapter 2: The mathematics of grand unified theory. Bentham Ebooks, 10-59. http://www.benthamscience.com/ebooks/9781608051786/index.htm

Escultura, E. E. (in press). The generalized integral as dual of Schwarz distribution. Journal of Nonlinear Studies.

Horgan, H. (1993). The death of proof. Scientific American, 5, 74-82.

Kiyosi, I. (Ed.), (1993). Encyclopedic dictionary of mathematics, corporate mathematical society of Japan. Chapter 5: The integers (2nd ed.). Cambridge, MA: MIT Press, 393-400.

Kline, M. (1980). Mathematics: The loss of certainty. Chapter 7: The axiom of choice (pp. 170-271). Oxford: Oxford University Press.

Lakatos, I. (1976). Proofs and refutations. Chapter 2: Counterexamples to Cauchy's proof of Euler's formula on the polyhedron (J. Worral \& E. Zahar Eds.). Cambridge: Cambridge University Press, 70-99.

Lakshmikantham, V., Escultura, E. E., \& Leela, S. (2009). The Hybrid Grand Unified Theory. Chapter 2: The mathematics of the HGUT. Paris: Atlantis (Elsevier Science, Ltd), 70-93.

Royden, H. L. (1983). Real analysis. Chapter 1: The real number system (3rd ed.). New York: MacMillan, 31-32.

Young, L. C. (1969). Lectures on the calculus of variations and optimal control theory. Volume II: The integrated Pontrjagin maximum principle. Philadelphia: W. B. Saunders, 410-498.

Young, L. C. (1980). Mathematicians and their times. Chapter 3: Some paradox. Amsterdam: North-Holland, 122-123. 